\title{
Reevaluation of Dystrophin Localization in the Mouse Retina
}

\author{
Eric Wersinger, ${ }^{1,2,3}$ Agnès Bordais, ${ }^{2}$ Yannick Schwab, ${ }^{4}$ Abdoulaye Sene, ${ }^{2,5}$ \\ Romain Bénard, ${ }^{2}$ Violaine Alunni, ${ }^{6}$ José-Alain Sabel, ${ }^{2,7,8,9}$ Alvaro Rendon, ,2 \\ and Michel J. Roux $x^{*, 1}$
}

Purpose. The roles of dystrophins in retinal physiology remain elusive. The lack of proper clustering of the potassium channel Kir4.1 and of the aquaporin AQP4 was proposed to be the basis of the ERG abnormality observed in many Duchenne muscular dystrophy (DMD) patients. However, the electroretinogram of Dp71-null mice, in which this clustering is disrupted, shows only a moderate reduction of the b-wave with no change in the implicit times. Additionally, the deficit in color discrimination found in DMD patients is hard to explain through the known expression of $D M D$ gene products. The authors thus decided to reexamine their distribution in the mouse retina.

Methods. Messenger RNA distribution was assessed by PCR coupled to laser microdissection of the outer and inner nuclear layers and by in situ hybridization for Dp427. Mouse retinas were double labeled for dystrophins versus presynaptic and postsynaptic proteins or antibodies specific for Dp427 or Dp427+Dp260.

RESUlTs. Messengers for Dp427, Dp260, and Dp140 were present in the inner nuclear layer. Dp 427 mRNA was further detected in bipolar cells and in some amacrine cells by in situ hybridization. Comparative labeling in wild-type and $m d x^{5 C v}$

From ${ }^{1}$ Translational Medicine and Neurogenetics, ${ }^{4}$ Imaging Center, and ${ }^{6}$ Microarrays, CNRS UMR 7104, Inserm U 964, Institut de Génétique et de Biologie Moléculaire et Cellulaire, Université de Strasbourg, Illkirch, France; ${ }^{2}$ Institut de la Vision, INSERM UMR_S968, CNRS UMR_7210, Université Pierre et Marie Curie Paris, Paris, France; ${ }^{7}$ Centre d'Investigation Clinique 503, Inserm-Centre Hospitalier National d'Ophtalmologie des Quinze-Vingts, Paris, France; ${ }^{8}$ Fondation Ophtalmologique Adolphe de Rothschild, Paris, France; and ${ }^{9}$ Institute of Ophthalmology, University College of London, London, United King dom.

Present affiliations: ${ }^{3}$ Sensorion Pharmaceuticals, Hopital St. Eloi, Montpellier, France; and ${ }^{5}$ Washington University School of Medicine, Department of Ophthalmology and Visual Sciences, St. Louis, Missouri.

Supported by Centre National de la Recherche Scientifique, INSERM, University Pierre and Marie Curie, Assistance Publique-Hôpitaux de Paris, Fédération des Aveugles de France, Retina-France, HFSP RGY0004/2003, Association Française contre les Myopathies, and the European Economic Community Grant RETRAINET HPRN-CT-2000 00098 .

Submitted for publication March 8, 2011; revised July 8 and August 5, 2011; accepted August 7, 2011.

Disclosure: E. Wersinger, None; A. Bordais, None; Y. Schwab, None; A. Sene, None; R. Bénard, None; V. Alunni, None; J.-A. Sahel, None; A. Rendon, None; M.J. Roux, None

*Each of the following is a corresponding author: Michel J. Roux, Translational Medicine and Neurogenetics, Institut de Génétique et de Biologie Moléculaire et Cellulaire, CNRS UMR 7104, INSERM U 964 , Université de Strasbourg, 1 rue Laurent Fries BP10142, 67404 Illkirch Cedex, France; mjroux@igbmc.fr.

Alvaro Rendon, Institut de la Vision, INSERM UMR_S968, CNRS UMR_7210, Université Pierre et Marie Curie Paris 06, 17 rue Moreau, Paris F-75012, France; alvaro.rendon@inserm.fr. retinas (lacking Dp427) indicated a differential distribution of Dp427 and Dp260 between rod and cone terminals.

Conclusions. In addition to their localization in photoreceptor terminals, Dp427, Dp260, and Dp140 are expressed in inner nuclear layer neurons, notably in bipolar cells for Dp427. Dp427 was proportionally more expressed in cone- than in rod-associated synapses compared with Dp260. (Invest Ophthalmol Vis Sci. 2011;52:7901-7908) DOI:10.1167/iovs.117519

$\mathrm{D}$ uchenne muscular dystrophy (DMD) and Becker muscular dystrophy (BMD) are severe X-linked myodegenerative diseases caused by mutations in the DMD gene and characterized by the defective expression of the full length dystrophin (Dp427). In addition to Dp427, the activation of internal promoters leads to the production of several shorter products, named Dp260, Dp140, Dp116, and Dp71 in reference to their estimated molecular weights. ${ }^{1-4}$ All but Dp116 were reported to be expressed in the retina. ${ }^{5-7} \mathrm{Dp} 427$, Dp260, and Dp140 were associated with the photoreceptor terminals and Dp71 in Müller glial cells and astrocytes.,

The complexes formed by dystrophins and associated proteins (DAPs), originally characterized to cluster acetylcholine receptors at the neuromuscular junction, appear to be equally important in the CNS because some DMD and BMD patients experience nonprogressive cognitive deficits, depending on the mutation position along the $D M D$ gene. ${ }^{9-12}$ Retinal physiology is also affected, with an abnormal scotopic electroretinogram (ERG), in which the b-wave presents both a strong reduction in amplitude, with a negative configuration, and increased implicit time. ${ }^{13-16}$ The photopic response is reduced as well, primarily in its ON component. ${ }^{17} \mathrm{~A}$ similar reduction of the ERG b-wave amplitude was found in the $m d x^{3 C v}$ mouse, deficient for all dystrophins, ${ }^{16}$ and in a $\beta$-dystroglycan mutant mouse. ${ }^{18}$ In these models, both the potassium channel Kir4.1 and the aquaporin AQP4 are mislocalized. ${ }^{8,19}$ Because potassium buffering by Müller cells was proposed to be the basis for the ERG b-wave, ${ }^{20,21}$ this delocalization was linked to the b-wave deficit. However, the electroretinogram of Dp71-null mice, in which Kir4.1 and AQP4 are similarly mislocalized, has been reported to have either a normal ${ }^{8}$ or a nonsignificantly reduced b-wave and nonaffected implicit times (Cia D, et al. IOVS 2010;51:ARVO E-abstract 4033), thus differing from those of DMD patients and $m d x^{3 C v}$ mice. Moreover, patients with mutations in Kir4.1 have a decreased sensitivity of their darkadapted ERG but normal b-wave amplitudes. ${ }^{22}$ The cause of the b-wave reduction in both humans and mouse models is thus still not understood. Moreover, a red-green color vision defect was recently reported in some DMD patients, ${ }^{23}$ which is hard to explain with the distribution of all dystrophins other than Dp71 restricted to photoreceptor terminals. Therefore, we aimed to reassess the distribution of dystrophin proteins in the 
TABle 1. Primary Antibodies

\begin{tabular}{lllcc}
\hline \multicolumn{1}{c}{ Target } & \multicolumn{1}{c}{ Type } & \multicolumn{1}{c}{ Source } & Dilution & Epitope \\
\hline Dystrophins & Mouse mAb & Novocastra NCL-DYS2 & $1 / 50$ & aa 3669-3685, human sequence \\
Dystrophins & Rabbit pAb & H4, gift from D. Mornet & $1 / 2000$ (light microscopy) & aa 3670-3685, human sequence \\
& & & $1 / 500$ (electron microscopy) & aa 1181-1388, human sequence \\
Dp427 & Mouse mAb & Novocastra NCL-DYS1 & $1 / 50$ & Recombinant protein corresponding to \\
Dp427 + Dp260 & Mouse mAb & C5G5, gift from D. Mornet ${ }^{25}$ & $1 / 50$ & mouse aa 1173-1728 \\
Calbindin & Rabbit pAb & Swant CB38a & $1 / 2000$ & $1 / 2000$ \\
mGluR6 & Rabbit pAb & Neuromics RA13105 & $1 / 3000$ & \\
NKCC & Mouse mAb & DSHB, University of Iowa, clone T4 & & \\
\hline
\end{tabular}

retina using a combination of laser capture microdissection combined with RT-PCR, in situ hybridization, and immunohistochemistry. We report here that the mRNAs coding for DMD gene products Dp427, Dp260, and Dp140 are expressed in both the outer nuclear layer (ONL) and the inner nuclear layer (INL). Dp427 mRNA is present in some bipolar cells (most probably from the ON subtype) and amacrine cells. By differential immunolabeling, we also show that Dp 427 is proportionally more expressed than Dp260 in synapses between cones and cone bipolar cells compared with those formed between rods and rod bipolar cells.

\section{Materials AND Methods}

\section{Animals}

C57BL/6J mice were purchased from Charles River (Les Oncins, France), and $m d x^{5 C v}$ was purchased from the Jackson Laboratory (Bar Harbor, ME). All animal experiments were conducted in compliance with the French Ministry of Agriculture and the European Community Council (directive no. 86/609/EEC, OJL 358, December 18, 1986) and the ARVO Statement for the Use of Animals in Ophthalmic and Vision Research.

\section{Immunohistochemistry}

In most cases, animals were killed by cervical dislocation. Eyes were opened along the ora serrata, and the eyecup was fixed 2 hours in PBS $+4 \%$ paraformaldehyde (PFA). After cryoprotection by immersion in a $30 \%$ sucrose PBS solution, eyecups were embedded in Shandon Cryomatrix (Anatomic Pathology International, Runcorn, UK), frozen on dry ice, and sliced at $14-\mu \mathrm{m}$ thickness in a cryostat (CM3000; Leica Microsystems GmbH, Wetzlar, Germany). For part of the data presented (see Figure 6), animals were anesthetized with intraperitoneal injection of ketamine $(70 \mathrm{mg} / \mathrm{kg})$ and xylazine $(5 \mathrm{mg} / \mathrm{kg})$ and were perfused with cold PBS and then with $4 \%$ PFA in cold PBS. The eyes were removed and the retinas were isolated before postfixation (2 hours in 4\% PFA) and then were included in 3\% agarose and sliced (50 $\mu \mathrm{m})$ with a vibratome (VT1000S; Leica). After blocking and permeabilization in PBS containing 5\% normal goat serum and $0.5 \%$ Triton $\mathrm{X}-100$, sections were incubated overnight at $4^{\circ} \mathrm{C}$ with primary antibodies in PBS containing 0.5\% Triton X-100. Primary antibodies (Table 1) were detected $\left(2\right.$ hours, $25^{\circ} \mathrm{C}$ ) using secondary goat anti-rabbit IgG antibodies coupled to Alexa Fluor 488 (Invitrogen, Cergy-Pontoise, France) or anti-mouse IgG coupled to Cy3 (Vector Laboratories, Bur- lingame, CA) diluted 1:500 in PBS containing 0.5\% Triton X-100. For clarity, dystrophin staining is shown in red in all merged confocal images, corresponding to double staining with other cellular markers. After mounting in medium (Vectashield; Vector Laboratories), the sections were imaged using a confocal laser scanning microscope (TCS SP2 AOBS; [Leica] and an HCX PL APO $63 \times / 1.32$ oil CS objective). ImageJ software (developed by Wayne Rasband, National Institutes of Health, Bethesda, MD; available at http://rsb.info.nih.gov/ij/index.html) was used to make maximal projections from selected planes of confocal stacks and to quantify the dystrophin staining (see Fig. 5 and Supplementary Fig. S4, http://www.iovs.org/lookup/suppl/doi:10.1167/ iovs.11-7519/-/DCSupplemental). Interpolation (bicubic, $\times 4$ ) (see Figs. 3C, 3D) was performed (Photoshop CS2; Adobe, San Jose, CA).

\section{Laser Microdissection}

Tissue Collection. Nonfixed eyes from three mice were embedded in Shandon Cryomatrix (Anatomic Pathology International), frozen on dry ice, and sliced at $10 \mu \mathrm{m}$ thickness in a cryostat (CM3000; Leica) mounted onto autoclaved membrane-coated slides specially designed for the laser microdissection microscope (LMD; PEN-Membrane, $2.0 \mu \mathrm{m}$; Microdissect GmbH, Mittenaar, Germany).

LMD Protocol and RNA Isolation. Slides were air dried, fixed in $70 \%$ ethanol for 2 minutes, washed in DEPC-water for 30 seconds, dipped in toluidine blue prepared in DEPC-water to facilitate the identification of the ONL and INL, dehydrated in graded ethanol solutions $(75 \%$ for 30 seconds, $95 \%$ for 30 seconds, $100 \%$ for 30 seconds), and placed in a vacuum desiccator for a minimum of 45 minutes. Laser capture was performed by separately cutting the ONL and INL from the membrane-coated slides with an LMD (LMD6000; Leica). Total RNA was extracted from the captured cells (RNeasy MicroKit; Qiagen, Courtabouf, France).

RT-PCR. The reverse transcription reaction was performed using a two-step RT-PCR kit (QuantiTect Rev. Transcription; Qiagen). Removal of genomic contamination using the DNA wipeout buffer provided by the kit was performed before reverse transcription. Only the extractions ( 4 of 6 ) passing the negative control in which reverse transcriptase was omitted were further processed (Supplementary Fig. S1A, http://www.iovs.org/lookup/suppl/doi:10.1167/iovs.117519/-/DCSupplemental). Amplification was performed in a total volume of $20 \mu \mathrm{L}$ containing $10 \mu \mathrm{L}$ master mix (including Taq DNA polymerase, reaction buffer, dNTP mix, and SYBR Green dye), $4 \mathrm{mM}$ $\mathrm{MgCl}_{2}, 0.25 \mu \mathrm{M}$ each primer (see list in Table 2), and $2 \mu \mathrm{L}$ cDNA template on a real-time PCR system (LightCycler; Roche Diagnostics,

Table 2. Primers Used for Amplification Following Laser Microdissection

\begin{tabular}{lll}
\hline \multicolumn{1}{c}{ Target } & \multicolumn{1}{c}{ Antisense Primer } & \multicolumn{1}{c}{ Sense Primer } \\
\hline Dp427 & CCC TTT GGA CCT GAT CTT GA & CAAA AGC AGC TGT TGC ATG GAT \\
Dp260 & CCA AGA AAA CCT TCA ACA GT & TGG ATA CTC TGT TCA AGC AA \\
Dp140 & ATG CAG TGA CTG AAG TGA AGA & GGA AGA AGG TAT CAG GAG TCC \\
mGluR6 & TCC AGT ACC AAG CAA CCA AT & CAC CAT CTT TTT TCC GTT CAC \\
Rhodopsin & TCC AGG TAC ATC CCA GAA GG & GCT GCC CAT AGC AGA AGA AG \\
\hline
\end{tabular}


Basel, Switzerland), with 40 cycles $\left(94^{\circ} \mathrm{C}\right.$ for 15 seconds, $55^{\circ} \mathrm{C}$ for 15 seconds, and $72^{\circ} \mathrm{C}$ for 25 seconds). Tm analysis was performed by increasing the temperature from $60^{\circ} \mathrm{C}$ to $90^{\circ} \mathrm{C}$ at a linear transition rate of $0.1^{\circ} \mathrm{C} / \mathrm{s}$. Aliquots $(5 \mu \mathrm{L})$ of each amplification reaction were separated on $2 \%$ agarose gels containing ethidium bromide.

\section{In Situ Hybridization}

Probe Synthesis. The Dp427 antisense (CTTTCAGGAAGATGACAGAATCAG) and control sense (ATTGTTCAGGGCATGAACTCTTGT) probes were digoxigenin-labeled riboprobes generated by in vitro transcription (Dig-UTP; Roche Diagnostics) from DNA templates using SP6 and T7 RNA polymerases (Promega, Madison, WI). The DNA templates were obtained by linearization of a plasmid (pGEM-T Easy; Promega) in which the probe sequence had been subcloned.

Tissue Preparation. Unfixed, whole eyes were embedded in resin (Shandon Cryomatrix; Anatomic Pathology International, Pittsburgh, PA) and frozen on dry ice. Cryosections (10-12 $\mu \mathrm{m})$ were collected on RNase-treated slides (Super-Frost Plus; Fisher Scientific, Illkirch, France) and stored at $-80^{\circ} \mathrm{C}$. Fixation, carried out in a staining system (Autostainer XL; Leica), was in 4\% PFA for 20 minutes at room temperature. Slides were then washed for 5 minutes in PBS, acetylated, and dried by passing them in successive ethanol solutions $(70 \%, 90 \%$, and $100 \%$ ).

In Situ Hybridization. The hybridization steps were performed using a liquid handling platform (Genesis Freedom EVO; Tecan Deutschland GmbH, Crailsheim, Germany). Briefly, slides carrying retinal sections were integrated into flow-through chambers positioned into the Tecan platform. After a first step of proteinase $\mathrm{K}$ digestion $(0.01 \mu \mathrm{g} / \mathrm{mL}$ in $50 \mathrm{mM}$ Tris, $5 \mathrm{mM}$ EDTA, $0.05 \%$ Tween-20, $\mathrm{pH} 8.0)$ in PBS, sections were prehybridized for 30 minutes in solution (HybMix; Ambion, Austin, TX) and then hybridized for 5 hours and 30 minutes in a humid chamber at $64^{\circ} \mathrm{C}$ in a solution consisting of the prehybridization solution with the addition of the digoxigenin-labeled RNA probe at a concentration of 300 to $600 \mathrm{ng} / \mathrm{mL}$. Sections were processed for the immunodetection of the digoxigenin label using an anti-digoxigenin antibody coupled to peroxidase (Roche Diagnostics) and revealed with a tyramine-biotin amplification reaction. The default amplification time was 45 minutes and was adjusted depending on the signal intensity. After the color reaction, sections were washed with different blocking solutions and coverslipped with hydromatrix (MicroTech-Laboratory, Graz, Austria).

\section{Results}

\section{Dystrophin mRNAs Are Expressed in Both INL and ONL}

To assess the expression of DMD gene products at the mRNA level in both the ONL and INL, LMD was used to extract RNA from selected retinal layers (Figs. 1A, 1B). To ensure the lack of contamination, the laser beam was systematically pointed on the first and last row of nuclei for each layer. RT-PCR coupled to the laser capture of three ONL or three to six INL collected from horizontal sections of the retina revealed that mRNAs from Dp427, Dp260, and Dp140 were expressed not only in the ONL, as expected, but also in the INL in three independent experiments (Fig. 1 and Supplementary Figs. S1B, S1C, http:// www.iovs.org/lookup/suppl/doi:10.1167/iovs.11-7519/-/DC Supplemental). Separation of PCR products showed bands at expected molecular weights (Figs. 1C, 1D). To ensure that the detection in the INL of these mRNAs was not due to the presence of genomic DNA or mRNA contamination from the ONL, we systematically tested our samples for the absence of the specific photoreceptor marker, rhodopsin, with primers amplifying a short region within exon 3 . The absence of rhodopsin in the INL samples, regardless of whether it was reverse transcribed, attested to the lack of contamination (Supplementary Fig. S1A, http://www.iovs.org/lookup/suppl/doi:10.1167/ iovs.11-7519/-/DCSupplemental).

\section{Dp427 mRNA Is Expressed in Some Bipolar and Amacrine Cells}

To explore the Dp427 mRNA expression pattern at the cellular level, we undertook in situ hybridization experiments using digoxigenin-labeled cRNA probes specific for Dp427. Unfortunately, the small sizes of the specific regions of Dp260 and Dp140 mRNAs did not allow the design of probes for these DMD gene products that we could validate. As a control, we tested the Dp427 antisense (Supplementary Figs. S2A-E, http://www.iovs.org/lookup/suppl/doi:10.1167/iovs.117519/-/DCSupplemental) and sense (Supplementary Figs. S2F-J, http://Www.iovs.org/lookup/suppl/doi:10.1167/ iovs.11-7519/-/DCSupplemental) probes on four tissues in which the Dp427 is known to be expressed. ${ }^{26}$ Although the sense probe was systematically negative (Supplementary Figs.
FIGURE 1. Dp427, Dp260, and Dp140 mRNAs are expressed in both the INL and the ONL. Vertical section of mouse retina stained with toluidine blue before (A) and after (B) laser capture of portions of the INL and ONL. PCR products obtained after RNA extraction from the ONL (C) and INL (D) using pairs of primers specific for the three longer forms of dystrophins, with bands at the expected lengths $(185,177$, and $110 \mathrm{bp}$ for Dp427, Dp260 and Dp140, respectively).
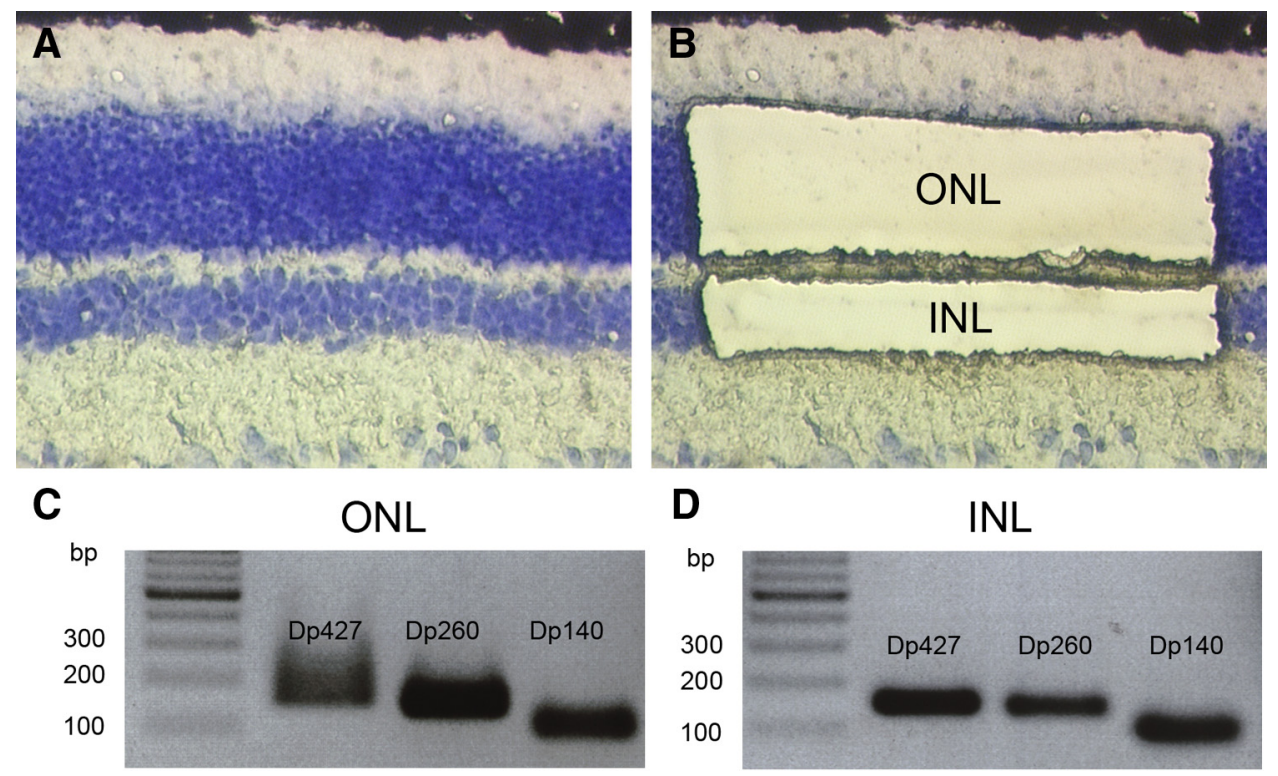


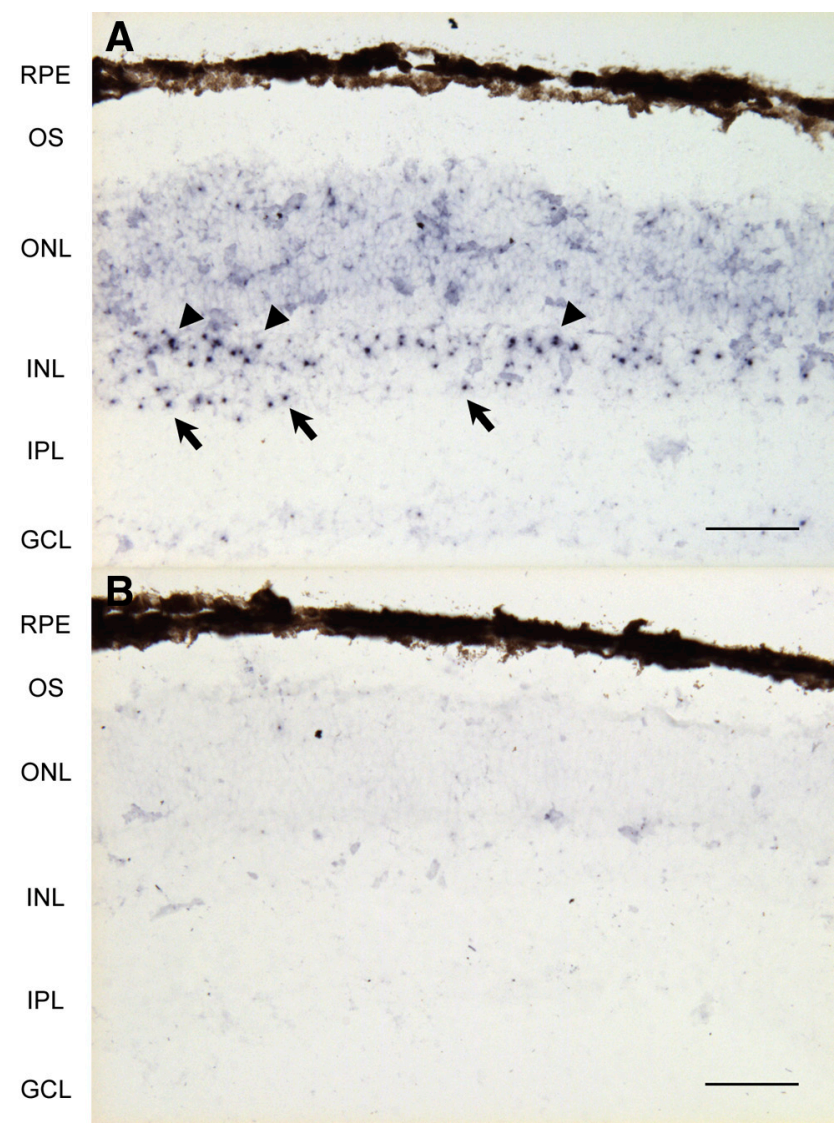

FIGURE 2. Dp 427 mRNA is present in some bipolar and putative amacrine cells. In situ hybridization with antisense (A) and sense (B) riboprobes for Dp427 mRNA in the mouse retina. Beyond the expected labeling of the ONL, Dp 427 mRNA is also present in many bipolar cells (arrowheads) and in some amacrine cells (arrows). Scale bars, $50 \mu \mathrm{m}$.

S2F-J, http://www.iovs.org/lookup/suppl/doi:10.1167/ iovs.11-7519/-/DCSupplemental), Dp427 mRNA was detected as expected in gastrocnemius muscle cells (Supplementary Fig. S2A, http://www.iovs.org/lookup/suppl/doi:10.1167/iovs.117519/-/DCSupplemental), in cerebellar Purkinje cells (Supplementary Figs. S2B, S2C, http://www.iovs.org/lookup/suppl/ doi:10.1167/iovs.11-7519/-/DCSupplemental), in the CA1 region of the hippocampus (Supplementary Fig. S2D, http:// www.iovs.org/lookup/suppl/doi:10.1167/iovs.11-7519/-/ DCSupplemental), and in the neocortex (Supplementary Fig. S2E, http://www.iovs.org/lookup/suppl/doi:10.1167/iovs.117519/-/DCSupplemental).

In the retina, Dp 427 mRNA signal could be observed in both the ONL and the INL, with a more intense signal in the INL than the ONL (Fig. 2A); the sense probe, however, did not give any signal (Fig. 2B) in three independent experiments. The weak signal in the ONL could have been due to the lower expression of Dp427 compared with Dp260 in the ONL, as suggested by the laser microdissection results (Fig. 1C). No signal was detected in the ganglion cell layer. In the INL, nearly all somas in the cell row next to OPL were labeled. As this row contains a large majority of rod bipolar cells ${ }^{27}$ and that OFF bipolar cell somas are found more toward the center of the INL, ${ }^{28}$ this indicates that most of the Dp427 expressing cells are ON bipolar cells, including most if not all rod bipolar cells. A significant signal was also detected in all experiments in the inner part of the INL, where amacrine cells are located (Fig. 2A, arrows). Some cell bodies in the central part of the INL, possibly from cone bipolar cells or amacrine cells, were also labeled. Thus, in addition to photoreceptors, at least two other retinal neuronal types expressed the full-length dystrophin.

\section{Dystrophin Proteins Localization in the Photoreceptor to Bipolar Cell Synapse}

In the OPL, expression of dystrophin proteins is considered in most species to be restricted to the photoreceptor terminal sub-domains that are wrapped around invaginated processes from bipolar cells, distal from the ribbon-containing active synaptic zones, notably through electron microscopy studies in mouse retina. ${ }^{29,30}$ An additional expression in the tip of bipolar cell dendrites was proposed only in the rabbit retina, as $\beta$-dystroglycan, a protein frequently associated with dystrophins, was detected in both photoreceptor terminals and bipolar cell dendrites by electron microscopy. ${ }^{31}$ The presence of Dp427 mRNA in bipolar cells reported here suggests that, in the mouse as well, these cells could contribute to the OPL immunostaining for dystrophin proteins. Such an expression might have been overlooked because of the difficulty separating it from the signal coming from photoreceptor terminals.

To investigate whether dystrophins were also localized in postsynaptic neuron tips, we labeled mouse retinal sections with antibodies directed against different markers of these cells. We first labeled horizontal cells with antibodies against calbindin. The terminals of horizontal cells were wrapped around the dystrophin puncta (corresponding to rod terminals), always on the ONL rather than the INL side (Fig. 3). A comparable situation was found at cone terminals, where calbindin staining was crossing the dystrophin bars in their weaker zones (the horizontal cell terminals were on the ONL side of the bar). This suggested that dystrophins were not expressed in horizontal cell tips entering photoreceptor terminals.

The metabotropic glutamate receptor mGluR6 expressed by ON bipolar cells is a good marker of a bipolar cell dendritic tip. ${ }^{32,33}$ At low magnification, it seemed to colocalize with dystrophin proteins, both in the OPL of wild-type mice and in ectopic synaptic contacts between photoreceptors and bipolar cells in the nob2 mouse retina. ${ }^{34}$ We labeled mouse retina slices with an antibody against mGluR6 and compared with the immunolabeling of dystrophin proteins at high magnification (Figs. 4A-C). When associated with rod terminals, the mGluR6 staining could be seen as pairs of parallel ingots, only partially colocalizing with dystrophin proteins. Indeed, the immunostaining for dystrophin proteins was not homogeneous but appeared as three bright parallel elements separated by two dimmer zones, corresponding to the position of the mGluR6 doublets (Fig. 4C). With retinal slices cut with an angle compared to the eye axis, the labeling for dystrophin proteins in

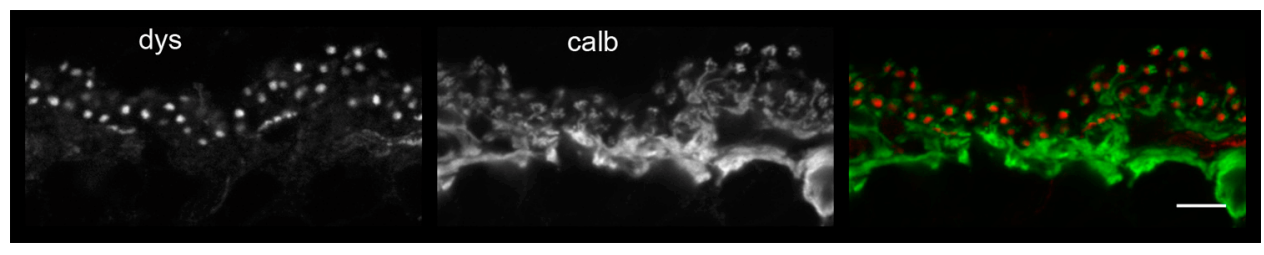

Figure 3. Dystrophins are not detected in horizontal cells. Vertical sections of mouse retina double stained for all dystrophins (dys, corresponding to dys 2 antibody; red in merged image) and the horizontal cell marker calbindin (calb; green in merged image). 
FIGURE 4. Partial colocalization of dystrophins and mGluR6. (A-C) Vertical sections of mouse retina double stained for all dystrophins (dys, corresponding to dys 2 antibody; red in merged images) and the mGluR6 receptor, expressed at the tips of ON bipolar cell dendrites. (A) Maximal projection of three consecutive confocal planes corresponding to a depth of $0.85 \mu \mathrm{m}$. (C) $50 \times 50$ pixel crop from a single confocal plane, interpolated to $200 \times 200$ pixels. (B) Two $128 \times 64$ pixel crops from two distinct confocal planes passing through a cone pedicle, interpolated to $512 \times 256$ pixels. Scale bars: $5 \mu \mathrm{m}$ (A); $1 \mu \mathrm{M}$ (B); $500 \mathrm{~nm}$ (C). (D, E) Interpretative schemes, indicating how the partial colocalization of dystrophins and mGluR6 labeling can be alternatively explained by the expression of dystrophins in both photoreceptor terminals and bipolar cell dendrites (D) or by the expression of dystrophins restricted to photoreceptor terminals $(\mathbf{E})$.

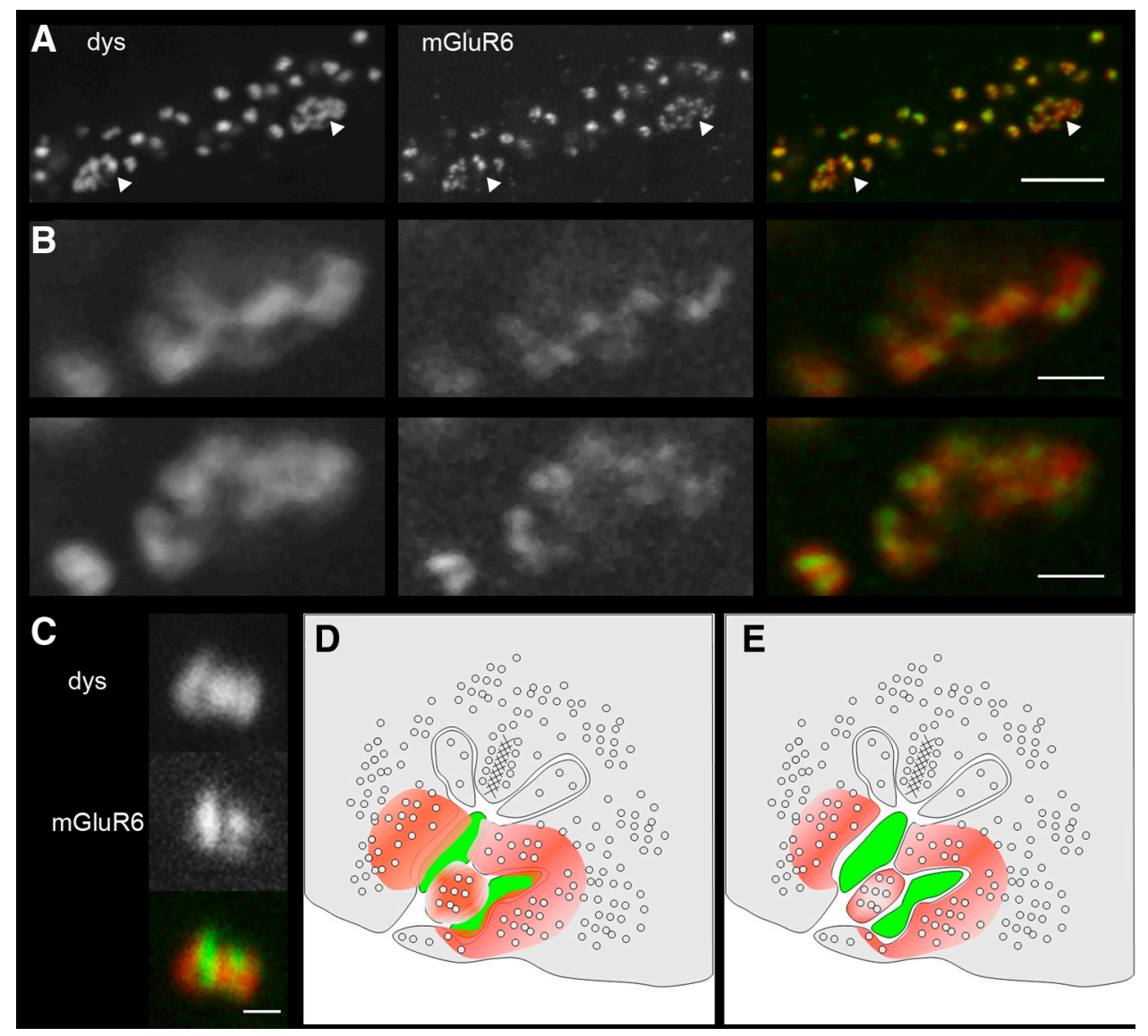

cones appeared not as bars but as "pretzels" covering a larger surface than the mGluR6 labeling (Fig. 4A, arrowheads). The discontinuities in the labeling for dystrophin proteins observed in rod terminals were not as evident in the cone terminals, resulting in a stronger colocalization with the mGluR6 labeling
(Fig. 4B). We used an antibody targeting another protein expressed in both rod and cone ON bipolar cells, the NKCC cation chloride cotransporter. ${ }^{35}$ Again, most NKCC-immunopositive dendritic tips were also labeled by antibody targeting dystrophin proteins, as shown by the superposition of the two
Figure 5. Cones have a higher Dp427/Dp260 ratio than rods. Vertical sections of wild-type $(\mathbf{A}, \mathbf{C})$ or $m d x^{5 C v}$ (B) mouse retina double stained in green for all dystrophins (dys, corresponding to $\mathrm{H} 4$ antibody) and in red for Dp427+ Dp260 (A, B; C5G5 antibody) or for Dp427 (C; dys1 antibody). (B) Because Dp427 was not expressed in the $m d x^{5 C v}$ mouse, the C5G5 labeling corresponded to Dp260 alone. Scale bars, $10 \mu \mathrm{m}$.

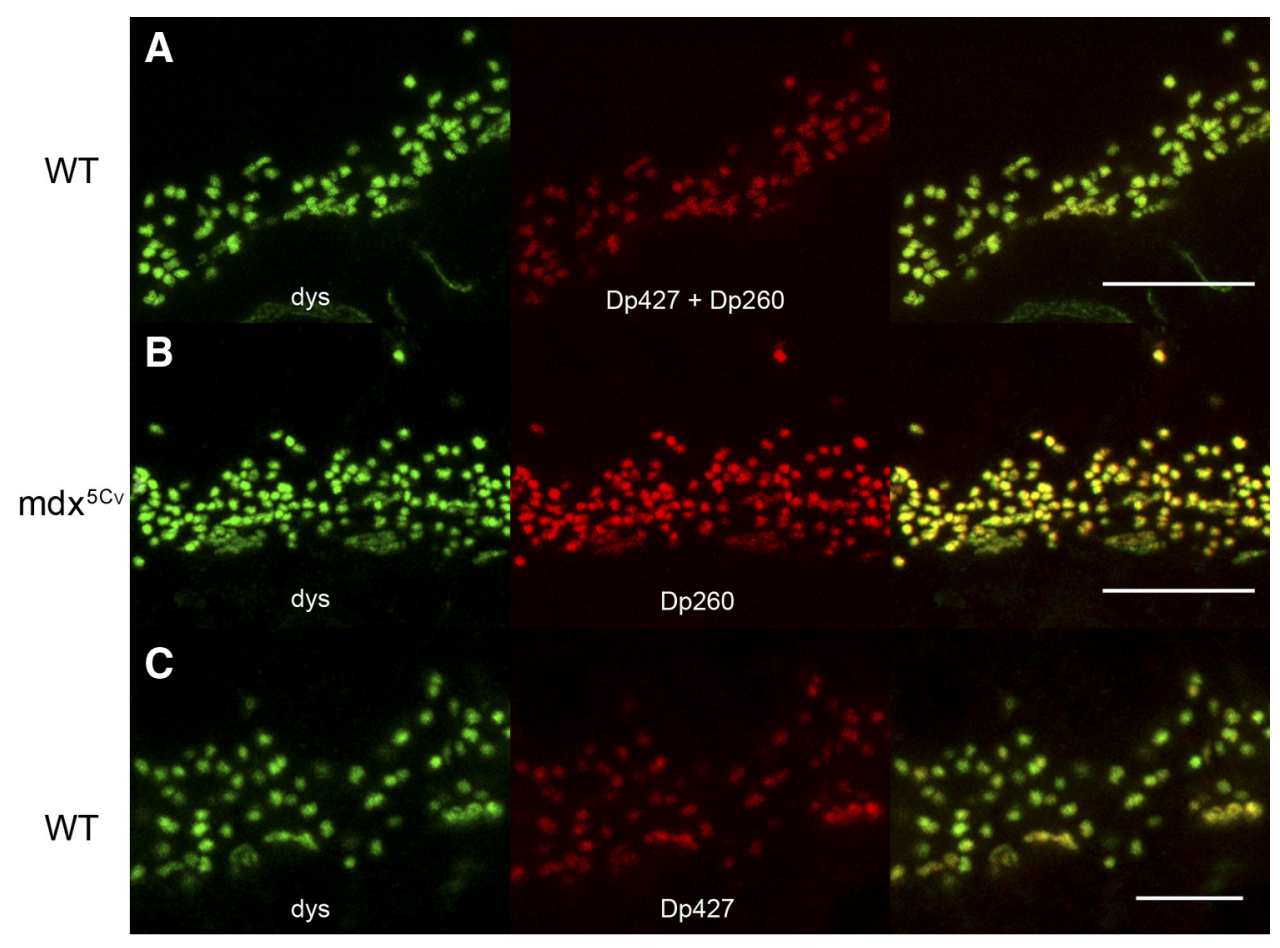


labelings (Supplementary Fig. S3, http://www.iovs.org/lookup/ suppl/doi:10.1167/iovs.11-7519/-/DCSupplemental). Together, these results suggest that dystrophins may be expressed postsynaptically in ON rod and cone bipolar cells, at a weaker level than in the surrounding photoreceptor elements (Fig. 4D). However, because of the small size of the dendritic tips, an artifactual mGluR6/dystrophin protein colocalization due to fluorescence diffusion from the intensely labeled photoreceptor terminals cannot be ruled out (Fig. 4E).

\section{Dp427 Is More Expressed in Cone- Than in Rod- Associated Synapses}

To get some insight into the relative distributions of the long dystrophin proteins in the OPL, we further compared the immunolabeling obtained with the pan-specific $\mathrm{H} 4$ antibody to those obtained with antibodies detecting either Dp427 (Dys1) or both Dp427 and Dp260 (C5G5) in wild-type and in $m d x^{5 C v}$ mice; this latter mouse strain lacked Dp427. As shown in Figure 5A, in the wild-type retina, both $\mathrm{H} 4$ and $\mathrm{C} 5 \mathrm{G} 5$ labeled cone and rod terminals with comparable intensities. The $\mathrm{H} 4$ intensity ratio (see Supplementary Methods and Supplementary Fig. S4, http://www.iovs.org/lookup/suppl/doi:10.1167/ iovs.11-7519/-/DCSupplemental) between cones and rods was 0.69 , whereas the ratio for C5G5 was 0.80 (measured on 25 spherules, 7 pedicles). To observe the distribution of Dp260, we labeled the $m d x^{5 C v}$ retina, in which $\mathrm{Dp} 427$ is not expressed, with the C5G5 antibody (Fig. 5B). As in the wild-type animals, rod terminals were more strongly stained with $\mathrm{H} 4$ and C5G5 than were cone terminals. The cone/rod ratio was 0.62 for $\mathrm{H} 4$ and 0.60 for $5 \mathrm{G} 5$ (10 spherules, 2 pedicles). When using the Dp427-specific Dys1 antibody, wild-type cone terminals were more intensely labeled than rod terminals (Fig. 5C); the cone/rod ratio was 0.68 for $\mathrm{H} 4$ and 1.21 for Dys 1 (15 spherules, 4 pedicles). Overall, this suggests that the density of the combined long forms of dystrophin (Dp427 + Dp260+ Dp140) was higher in rod than in cone terminals but that Dp427 was more expressed in cones than in rods.

\section{Dystrophins Are Expressed in OFF Bipolar Cells, Amacrine Cells, or Both}

Detection of Dp427 mRNA in amacrine cells suggests that Dp427 should be expressed in the inner retina and not only in the OPL. Similarly, the expression of Dp427 in bipolar cells may not be restricted to their dendrites. Dp260 and Dp140 could also be expressed outside the OPL. We thus looked in more detail at the inner nuclear labeling obtained with various anti-dystrophin antibodies. The monoclonal ones used in this study, either the pan-dystrophin Dys2 or the more specific Dys1 (Dp427) or C5G5 (Dp427 and Dp260), did not stain structures in the INL/inner plexiform layer (IPL) aside from the blood vessels for Dys2 as expected from the known Dp71 distribution. $^{8}$ On the other hand, the polyclonal $\mathrm{H} 4$ antibody labeled the base of the main neurite of inner nuclear neurons, which through their localization are most likely amacrine cells or OFF bipolar cells (Figs. 6A, 6C), and faintly labeled the IPL, primarily in its half closer to the INL (Fig. 6A). Both types of label were not detected in $m d x^{3 C v}$ retinal slices, incubated in the same well as the wild-type slices (Figs. 6B, 6D).

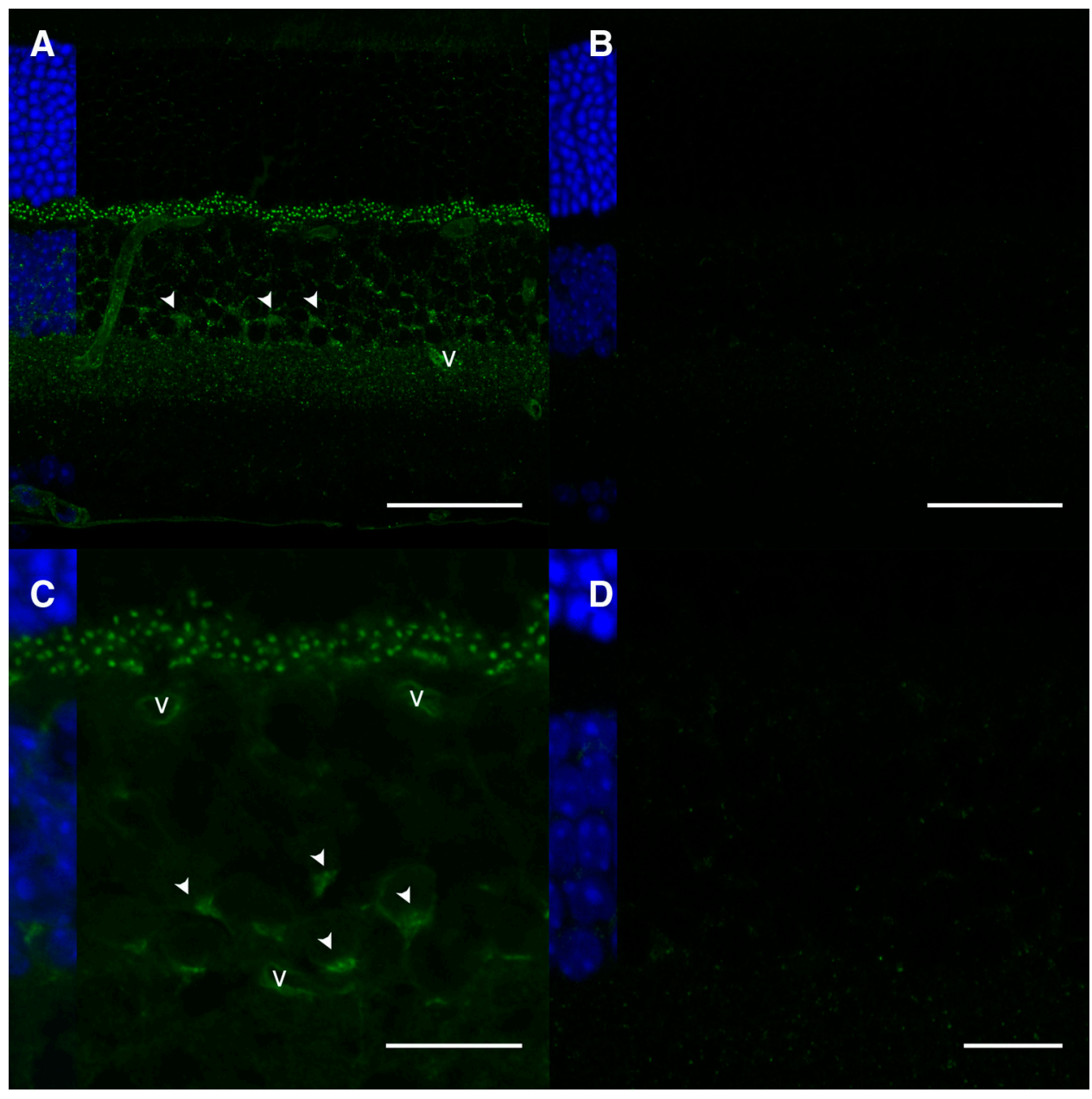

FIGURE 6. Dystrophins are expressed in putative OFF bipolar and amacrine cells. Vertical sections of wild-type $(\mathbf{A}, \mathbf{C})$ or $m d x^{3 C v}(\mathbf{B}, \mathbf{D})$ mouse retina stained for all dystrophins (green, corresponding to the H4 antibody). (A, B) To ensure a valid comparison, wild-type and $m d x^{3 C v}$ sections were processed in parallel in the same well for immunohistochemistry, and confocal acquisitions were performed with the same settings and at the same depth in the slice, based on the DAPI signal (represented to the left of each section). Labeled structures in inner nuclear neurons, at the base of their main neurite, are indicated by arrowheads; blood vessels are indicated by $v$. Images are projections of four (A, B, D) or five (C) successive confocal planes, corresponding to thicknesses of 1.59 and $1.14 \mu \mathrm{m}$, respectively. Scales bars: $50 \mu \mathrm{m}$ (A, B); $15 \mu \mathrm{m}(\mathbf{C}, \mathbf{D})$. 


\section{Discussion}

Previous light and electron microscopy studies on mammalian retinas have proposed that dystrophins, sometimes indirectly through the localization of $\beta$-dystroglycan, were expressed exclusively in photoreceptor terminals, ${ }^{6,29-31,36-38}$ with the exception of the rabbit retina, for which a postsynaptic localization of $\beta$-dystroglycan in horizontal and bipolar cells was detected. $^{31}$ Here we provide evidence that, in the mouse retina, the DMD gene products other than Dp71 are expressed not only in photoreceptors but also in the INL. Notably, the full-length dystrophin Dp427 mRNA was detected in ON bipolar cells and in some amacrine cells, and dystrophin immunolabeling was detected in cells close to the IPL, most probably amacrine or OFF bipolar cells.

Our results suggest that Dp260 mRNA is the most expressed form in the ONL, followed by the Dp140 and the Dp427 mRNA, whereas in the INL, Dp427 mRNA was more expressed than Dp140 and Dp260. This is in good agreement with our previous results from the rat retina regarding the relative expression in the ONL. In the rat however, Dp260 mRNA was exclusively expressed in the outer retina. ${ }^{39}$ For Dp 427 , the in situ hybridization data suggest that it is expressed mostly in rod bipolar cells and in some amacrine cells.

Coimmunostaining for dystrophins and proteins expressed in the tips of ON bipolar cells (mGluR6, NKCC) suggested colocalization. However, at high magnification, the dystrophin staining was not homogeneous, and its weaker zones matched those of the mGluR6 or NKCC staining. The combination of the invaginated structure and small size of the dendritic tips thus precludes drawing a solid conclusion from confocal microscopy. Images from Figure 4 and Supplementary Figure S3 (http://www.iovs.org/lookup/suppl/doi:10.1167/iovs.11-7519/-/ DCSupplemental) can alternatively correspond to an expression of dystrophins in the dendrites of ON bipolar cells (Fig. 4D) or to a dystrophin expression strictly in the photoreceptor terminals (Fig. 4E).

Another indirect argument for the expression of dystrophin proteins in INL neurons is the in situ hybridization data for $\beta$-dystroglycan reported by Montanaro et al., ${ }^{40}$ showing strong expression in the INL that was partially reduced in the mdx strain, which lacks Dp427. Together with the higher Dp427/ Dp260 ratio and the higher colocalization of mGluR6 with dystrophin proteins, observed in the cone-associated synaptic zones compared with rod zones, we suggest that Dp427 could be expressed in cone ON bipolar cell dendrites. New imaging techniques resulting in better resolution, such as STED and PALM microscopy, as well as the more conventional electron microscopy should help answer this issue. We have tried both pre-embedding and post-embedding immunohistochemistry, including on ultrathin cryosections, in which the embedding resin is replaced by a high-sucrose solution to increase epitope accessibility. ${ }^{41}$ As in previous reports from other groups, ${ }^{29,31,37}$ our pre-embedding results indicated expression strictly in photoreceptor terminals (Supplementary Figs. S5A, S5B, http://Www.iovs.org/lookup/suppl/doi: 10.1167/iovs.11-7519/-/DCSupplemental). However, the geometry of the synapse might have hindered the access of the antibody to the postsynaptic structures. When observed with electron microscopy after fluorescence immunohistochemistry treatment, the tips of bipolar dendrites were preserved compared with the surrounding photoreceptor terminal structures, suggesting that it was not as accessible to the detergent and thus not as well permeabilized as presynaptic elements (data not shown). Because the DAB reaction affected the tissue structure, longer revelation time could not be used to compensate for this bias. With the Tokuyasu technique, only a few gold particles could be detected per terminal. The poor contrast prevented a definitive identification of bipolar dendrites (Supplementary Figs. S5C, S5D, http://www.iovs.org/lookup/suppl/doi:10.1167/ iovs.11-7519/-/DCSupplemental). Thus, though the expression of Dp427 mRNA in at least part of ON bipolar cells is established, the expression of dystrophin proteins in these cells remains to be demonstrated.

It has recently been reported that some DMD patients, with mutations affecting Dp260, present a deficit in red-green discrimination. $^{23}$ Such a deficit is difficult to reconcile with a Dp260 expression restricted to photoreceptor terminals because its inactivation should affect in a similar way the output of the various cone types. We have reported here that Dp427 mRNA is expressed in some amacrine cells and that Dp260 and Dp140 mRNA are expressed in the INL. Dystrophin immunoreactivity was located at the base of the main neurite of some of the neurons in the two rows closer to the IPL, suggesting that they were OFF bipolar or amacrine cells, and more diffusely in the outer part of the INL. Dystrophins may be expressed differentially between retinal subnetworks, notably in the mouse equivalent to those involved in color processing in primates.

\section{References}

1. Bar S, Barnea E, Levy Z, Neuman S, Yaffe D, Nudel U. A novel product of the Duchenne muscular dystrophy gene which greatly differs from the known isoforms in its structure and tissue distribution. Biochem J. 1990;272:557-560.

2. Byers TJ, Lidov HG, Kunkel LM. An alternative dystrophin transcript specific to peripheral nerve. Nat Genet. 1993;4:77-81.

3. D'Souza VN, Nguyen TM, Morris GE, Karges W, Pillers DA, Ray PN. A novel dystrophin isoform is required for normal retinal electrophysiology. Hum Mol Genet. 1995;4:837-842.

4. Lidov HG, Selig S, Kunkel LM. Dp140: a novel 140 kDa CNS transcript from the dystrophin locus. Hum Mol Genet. 1995;4: 329-335.

5. Howard PL, Dally GY, Wong MH, et al. Localization of dystrophin isoform Dp71 to the inner limiting membrane of the retina suggests a unique functional contribution of Dp71 in the retina. Hum Mol Genet. 1998;7:1385-1391.

6. Ueda H, Baba T, Terada N, Kato Y, Tsukahara S, Ohno S. Dystrophin in rod spherules; submembranous dense regions facing bipolar cell processes. Histochem Cell Biol. 1997;108:243-248.

7. Rodius F, Claudepierre T, Rosas-Vargas H, et al. Dystrophins in developing retina: Dp260 expression correlates with synaptic maturation. Neuroreport. 1997;8:2383-2387.

8. Dalloz C, Sarig R, Fort P, et al. Targeted inactivation of dystrophin gene product Dp71: phenotypic impact in mouse retina. $\mathrm{Hum} \mathrm{Mol}$ Genet. 2003;12:1543-1554.

9. Billard C, Gillet P, Signoret JL, et al. Cognitive functions in Duchenne muscular dystrophy: a reappraisal and comparison with spinal muscular atrophy. Neuromuscul Disord. 1992;2:371-378.

10. Cyrulnik SE, Fee RJ, De Vivo DC, Goldstein E, Hinton VJ. Delayed developmental language milestones in children with Duchenne's muscular dystrophy. J Pediatr. 2007;150:474-478.

11. Lenk U, Hanke R, Thiele H, Speer A. Point mutations at the carboxy terminus of the human dystrophin gene: implications for an association with mental retardation in DMD patients. $\mathrm{Hum} \mathrm{Mol}$ Genet. 1993;2:1877-1881.

12. Muntoni F, Torelli S, Ferlini A. Dystrophin and mutations: one gene, several proteins, multiple phenotypes. Lancet Neurol. 2003; 2:731-740.

13. Cibis GW, Fitzgerald KM, Harris DJ, Rothberg PG, Rupani M. The effects of dystrophin gene mutations on the ERG in mice and humans. Invest Opbthalmol Vis Sci. 1993;34:3646-3652.

14. Sigesmund DA, Weleber RG, Pillers DA, et al. Characterization of the ocular phenotype of Duchenne and Becker muscular dystrophy. Opbthalmology. 1994;101:856-865.

15. Pillers DA, Fitzgerald KM, Duncan NM, et al. Duchenne/Becker muscular dystrophy: correlation of phenotype by electroretinog- 
raphy with sites of dystrophin mutations. Hum Genet. 1999;105: 2-9.

16. Pillers DA, Bulman DE, Weleber RG, et al. Dystrophin expression in the human retina is required for normal function as defined by electroretinography. Nat Genet. 1993;4:82-86.

17. Fitzgerald KM, Cibis GW, Giambrone SA, Harris DJ. Retinal signal transmission in Duchenne muscular dystrophy: evidence for dysfunction in the photoreceptor/depolarizing bipolar cell pathway. J Clin Invest. 1994;93:2425-2430.

18. Satz JS, Philp AR, Nguyen H, et al. Visual impairment in the absence of dystroglycan. J Neurosci. 2009;29:13136-13146.

19. Connors NC, Kofuji P. Dystrophin Dp71 is critical for the clustered localization of potassium channels in retinal glial cells. J Neurosci. 2002;22:4321- 4327 .

20. Miller RF, Dowling JE. Intracellular responses of the Müller (glial) cells of mudpuppy retina: their relation to b-wave of the electroretinogram. J Neurophysiol. 1970;33:323-341.

21. Newman EA, Odette LL. Model of electroretinogram b-wave generation: a test of the K+ hypothesis. J Neurophysiol. 1984;51: $164-182$.

22. Thompson DA, Feather S, Stanescu HC, et al. Altered electroretinograms in patients with KCNJ10 mutations and EAST syndrome. J Physiol. 2011;589:1681-1689.

23. Costa MF, Oliveira AGF, Feitosa-Santana C, Zatz M, Ventura DF. Red-green color vision impairment in Duchenne muscular dystrophy. Am J Hum Genet. 2007;80:1064-1075.

24. Royuela M, Chazalette D, Hugon G, et al. Formation of multiple complexes between beta-dystroglycan and dystrophin family products. J Muscle Res Cell Motil. 2003;24:387-397.

25. Fabbrizio E, Latouche J, Rivier F, Hugon G, Mornet D. Re-evaluation of the distributions of dystrophin and utrophin in sciatic nerve. Biochem J. 1995;312 (pt 1):309-314.

26. Górecki DC, Monaco AP, Derry JM, Walker AP, Barnard EA, Barnard PJ. Expression of four alternative dystrophin transcripts in brain regions regulated by different promoters. Hum Mol Genet. 1992;1:505-510.

27. Haverkamp S, Wässle $H$. Immunocytochemical analysis of the mouse retina. J Comp Neurol. 2000;424:1-23.

28. Ghosh KK, Bujan S, Haverkamp S, Feigenspan A, Wässle H. Types of bipolar cells in the mouse retina. J Comp Neurol. 2004;469:70 82
29. Blank M, Koulen P, Blake DJ, Kröger S. Dystrophin and betadystroglycan in photoreceptor terminals from normal and $\mathrm{mdx} 3 \mathrm{Cv}$ mouse retinae. Eur J Neurosci. 1999;11:2121-2133.

30. Ueda H, Kato Y, Baba T, et al. Immunocytochemical study of dystrophin localization in cone cells of mouse retinas. Invest Ophthalmol Vis Sci. 1997;38:1627-1630.

31. Koulen P, Blank M, Kröger S. Differential distribution of betadystroglycan in rabbit and rat retina. J Neurosci Res. 1998;51:735747.

32. Nomura A, Shigemoto R, Nakamura Y, Okamoto N, Mizuno N, Nakanishi S. Developmentally regulated postsynaptic localization of a metabotropic glutamate receptor in rat rod bipolar cells. Cell. 1994;77:361-369.

33. Vardi N. Alpha subunit of Go localizes in the dendritic tips of ON bipolar cells. J Comp Neurol. 1998;395:43-52.

34. Bayley PR, Morgans CW. Rod bipolar cells and horizontal cells form displaced synaptic contacts with rods in the outer nuclear layer of the nob2 retina. J Comp Neurol. 2007;500:286-298.

35. Vardi N, Zhang LL, Payne JA, Sterling P. Evidence that different cation chloride cotransporters in retinal neurons allow opposite responses to GABA. J Neurosci. 2000;20:7657-7663.

36. Ueda H, Gohdo T, Ohno S. Beta-dystroglycan localization in the photoreceptor and Müller cells in the rat retina revealed by immunoelectron microscopy. J Histochem Cytochem. 1998;46:185-191.

37. Ueda H, Kobayashi T, Mitsui K, Tsurugi K, Tsukahara S, Ohno S Dystrophin localization at presynapse in rat retina revealed by immunoelectron microscopy. Invest Ophthalmol Vis Sci. 1995;36: 2318-2322

38. Schmitz F, Drenckhahn D. Localization of dystrophin and betadystroglycan in bovine retinal photoreceptor processes extending into the postsynaptic dendritic complex. Histochem Cell Biol. 1997;108:249-255.

39. Claudepierre T, Rodius F, Frasson M, et al. Differential distribution of dystrophins in rat retina. Invest Ophthalmol Vis Sci. 1999;40: $1520-1529$

40. Montanaro F, Carbonetto S, Campbell KP, Lindenbaum M. Dystroglycan expression in the wild type and $\mathrm{mdx}$ mouse neural retina: synaptic colocalization with dystrophin, dystrophin-related protein but not laminin. J Neurosci Res. 1995;42:528-538.

41. Tokuyasu KT. A technique for ultracryotomy of cell suspensions and tissues. J Cell Biol. 1973;57:551-565. 